\title{
OBSERVATION OF THE TeV GAMMA-RAY SOURCE MGRO J1908+06 WITH ARGO-YBJ
}

\author{
B. Bartoli ${ }^{1,2}$, P. Bernardini ${ }^{3,4}$, X. J. Bi ${ }^{5}$, C. Bleve ${ }^{3,4}$, I. Bolognino $^{6,7}$, P. Branchini $^{8}$, A. Budano ${ }^{8}$,
} A. K. Calabrese Melcarne ${ }^{9}$, P. Camarri ${ }^{10,11}$, Z. CaO ${ }^{5}$, R. Cardarelli ${ }^{11}$, S. Catalanotti ${ }^{1,2}$, C. Cattaneo ${ }^{7}$, S. Z. Chen ${ }^{5}$, T. L. $\mathrm{Chen}^{12}$, Y. $\mathrm{CheN}^{5}$, P. Creti ${ }^{4}$, S. W. Cui ${ }^{13}$, B. Z. Dai ${ }^{14}$, G. D'Alí Staiti ${ }^{15,16}$, DanZengluobu ${ }^{12}$, M. Dattoli ${ }^{17,18,19}$, I. De Mitri ${ }^{3,4}$, B. D’Ettorre Piazzoli ${ }^{1,2}$, T. Di Girolamo ${ }^{1,2}$, X. H. Ding ${ }^{12}$, G. Di Sciascio ${ }^{11}$, C. F. Feng $^{20}$, Zhaoyang Feng ${ }^{5}$, Zhenyong Feng ${ }^{21}$, F. Galeazzi ${ }^{8}$, E. Girolettio ${ }^{6,7}$, Q. B. Gou ${ }^{5}$, Y. Q. Guo ${ }^{5}$, H. H. He ${ }^{5}$, Haibing Hu ${ }^{12}$, Hongbo $\mathrm{Hu}^{5}$,

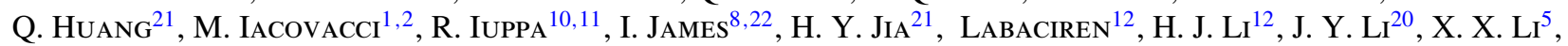

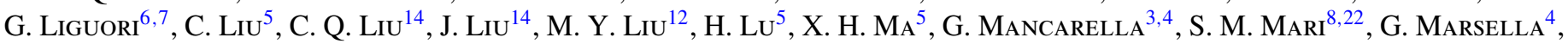
D. Martello ${ }^{3,4}$, S. Mastroianni ${ }^{2}$, P. Montini ${ }^{8,22}$, C. C. Ning $^{12}$, A. Pagliaro ${ }^{16,23}$, M. Panareo ${ }^{4}$, B. Panico ${ }^{10,11}$, L. Perrone $^{4}$,

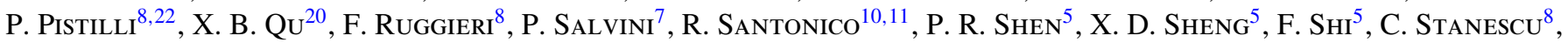

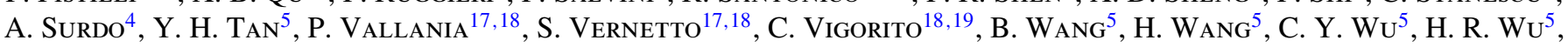

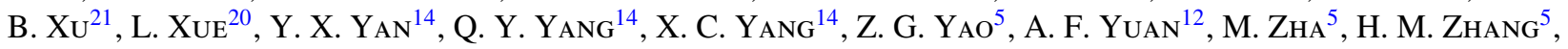

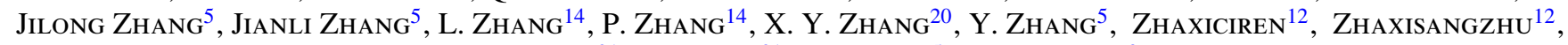
X. X. ZHOU ${ }^{21}$, F. R. ZHU ${ }^{21}$, Q. Q. ZHU ${ }^{5}$, AND G. ZiZZI ${ }^{9}$

(The Argo-YBJ Collaboration)

${ }^{1}$ Dipartimento di Fisica dell'Università di Napoli “Federico II," Complesso Universitario di Monte Sant'Angelo, via Cinthia, 80126 Napoli, Italy

${ }^{2}$ Istituto Nazionale di Fisica Nucleare, Sezione di Napoli, Complesso Universitario di Monte Sant'Angelo, via Cinthia, 80126 Napoli, Italy

${ }^{3}$ Dipartimento di Matematica e Fisica "Ennio De Giorgi," Università del Salento, via per Arnesano, 73100 Lecce, Italy

${ }^{4}$ Istituto Nazionale di Fisica Nucleare, Sezione di Lecce, via per Arnesano, 73100 Lecce, Italy

${ }^{5}$ Key Laboratory of Particle Astrophysics, Institute of High Energy Physics, Chinese Academy of Sciences, P.O. Box 918, 100049 Beijing, China

${ }^{6}$ Dipartimento di Fisica Nucleare e Teorica dell'Università di Pavia, via Bassi 6, 27100 Pavia, Italy

${ }^{7}$ Istituto Nazionale di Fisica Nucleare, Sezione di Pavia, via Bassi 6, 27100 Pavia, Italy

${ }^{8}$ Istituto Nazionale di Fisica Nucleare, Sezione di Roma Tre, via della Vasca Navale 84, 00146 Roma, Italy

${ }^{9}$ Istituto Nazionale di Fisica Nucleare-CNAF, Viale Berti-Pichat 6/2, 40127 Bologna, Italy

${ }^{10}$ Dipartimento di Fisica dell'Università di Roma “Tor Vergata," via della Ricerca Scientifica 1, 00133 Roma, Italy

${ }^{11}$ Istituto Nazionale di Fisica Nucleare, Sezione di Roma Tor Vergata, via della Ricerca Scientifica 1, 00133 Roma, Italy

12 Tibet University, 850000 Lhasa, Xizang, China

${ }^{13}$ Hebei Normal University, Shijiazhuang 050016, Hebei, China

${ }^{14}$ Yunnan University, 2 North Cuihu Rd., 650091 Kunming, Yunnan, China

${ }^{15}$ Dipartimento di Fisica, Università degli Studi di Palermo, Viale delle Scienze, Edificio 18, 90128 Palermo, Italy

${ }^{16}$ Istituto Nazionale di Fisica Nucleare, Sezione di Catania, Viale A. Doria 6, 95125 Catania, Italy

${ }^{17}$ Osservatorio Astrofisico di Torino dell'Istituto Nazionale di Astrofisica, corso Fiume 4, 10133 Torino, Italy

${ }^{18}$ Istituto Nazionale di Fisica Nucleare, Sezione di Torino, via P. Giuria 1, 10125 Torino, Italy

${ }^{19}$ Dipartimento di Fisica dell'Università di Torino, via P. Giuria 1,10125 Torino, Italy

${ }^{20}$ Shandong University, 250100 Jinan, Shandong, China

${ }^{21}$ Southwest Jiaotong University, 610031 Chengdu, Sichuan, China

22 Dipartimento di Fisica dell’Università “Roma Tre," via della Vasca Navale 84, 00146 Roma, Italy

${ }^{23}$ Istituto di Astrofisica Spaziale e Fisica Cosmica dell'Istituto Nazionale di Astrofisica, via La Malfa 153, 90146 Palermo, Italy Received 2012 March 8; accepted 2012 July 20; published 2012 November 14

\begin{abstract}
The extended gamma-ray source MGRO J1908+06, discovered by the Milagro air shower detector in 2007, has been observed for $\sim 4$ years by the ARGO-YBJ experiment at TeV energies, with a statistical significance of 6.2 standard deviations. The peak of the signal is found at a position consistent with the pulsar PSR J1907+0602. Parameterizing the source shape with a two-dimensional Gauss function, we estimate an extension of $\sigma_{\text {ext }}=0.49 \pm$ 0.22 , which is consistent with a previous measurement by the Cherenkov Array H.E.S.S. The observed energy

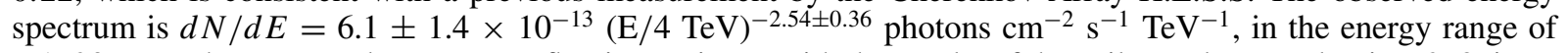
$\sim 1-20 \mathrm{TeV}$. The measured gamma-ray flux is consistent with the results of the Milagro detector, but is $\sim 2-3$ times larger than the flux previously derived by H.E.S.S. at energies of a few TeV. The continuity of the Milagro and ARGO-YBJ observations and the stable excess rate observed by ARGO-YBJ and recorded in four years of data support the identification of MGRO J1908+06 as the steady powerful TeV pulsar wind nebula of PSR J1907+0602, with an integrated luminosity over $1 \mathrm{TeV} \sim 1.8$ times the luminosity of the Crab Nebula.
\end{abstract}

Key words: gamma rays: general - pulsars: individual (MGRO J1908+06)

Online-only material: color figures

\section{INTRODUCTION}

The Galactic gamma-ray source MGRO J1908+06 was discovered by the Milagro air shower detector in a survey of the Galactic plane at a median energy of $\sim 20 \mathrm{TeV}$ (Abdo et al. 2007). The data were consistent both with a point source and with an extended source with a diameter of $<2.6$.
Assuming a spectrum of $\propto E^{-2.3}$, the measured flux at the median energy of $20 \mathrm{TeV}$ is $8.8 \pm 2.4 \times 10^{-15}$ photons $\mathrm{cm}^{-2}$ $\mathrm{s}^{-1} \mathrm{TeV}^{-1}$.

A marginal detection of a source consistent with the position of MGRO J1908+06 was already reported by the Tibet AS- $\gamma$ array (Zhang 2003), but not confirmed in a more recent paper (Amenomori et al. 2010). 
The source was later observed by the H.E.S.S. (Aharonian et al. 2009) and VERITAS (Ward 2008) Cherenkov telescopes. In particular, H.E.S.S detected an extended source (HESS J1908+063) at energies above $300 \mathrm{GeV}$ (with statistical significance of $\sim 11$ standard deviations) positionally consistent with MGRO J1908+06. The measured source extension, evaluated assuming a symmetrical two-dimensional Gaussian shape, was $\sigma_{\text {ext }}=0.34_{-0.03}^{+0.04}$.

H.E.S.S. reported a power-law differential energy spectrum with a photon index of $2.10 \pm 0.07_{\text {stat }} \pm 0.2_{\text {sys }}$ in the energy range of $0.3-20 \mathrm{TeV}$, and a flux at $1 \mathrm{TeV}$ of $\left(4.14 \pm 0.32_{\text {stat }} \pm\right.$ $\left.0.83_{\text {sys }}\right) \times 10^{-12}$ photons $\mathrm{cm}^{-2} \mathrm{~s}^{-1} \mathrm{TeV}^{-1}$. The integrated flux above $1 \mathrm{TeV}$ is $17 \%$ that of the Crab Nebula.

After the release of the Bright Source List by the Fermi collaboration (Abdo et al. 2009a), Milagro reported the association of MGRO J1908+06 to the LAT pulsar 0FGL J1907.5+0602 (later renamed PSR J1907+0602), pulsating with a period of $106.6 \mathrm{~ms}$ (Abdo et al. 2009b). The peak of the Milagro emission was 0.3 off the pulsar, but consistent with the pulsar location within the measurement error (0.27). Assuming a spectrum of $\propto E^{-2.6}$, Milagro reported a flux of $116.7 \pm 15.8 \times 10^{-17}$ photons $\mathrm{cm}^{-2} \mathrm{~s}^{-1} \mathrm{TeV}^{-1}$, at the median energy of $35 \mathrm{TeV}$.

The association of MGRO J1908+06 with PSR J1907+0602 was also supported in Abdo et al. (2010), where a multiwavelength study of the pulsar and the surrounding region has been performed with radio, X-ray and Fermi gamma-ray data. Because of the small angular distance between the pulsar and the centroid of the H.E.S.S. extended source, the authors argue that the latter is plausibly a wind nebula of the pulsar.

Performing an off-pulse measurement, Fermi sets an upper limit to the HESS J1908+063 flux in the energy region $0.1-25 \mathrm{GeV}$, suggesting that the spectrum has a low-energy turnover between $20 \mathrm{GeV}$ and $300 \mathrm{GeV}$. With radio and X-ray data, a lower limit to the pulsar distance was set to $\sim 3.2 \mathrm{kpc}$, deriving for the nebula a physical size $\geqslant 40 \mathrm{pc}$.

Later, Milagro evaluated the energy spectrum of the source in the 2-100 $\mathrm{TeV}$ region, reporting a hard power-law spectrum with an exponential cutoff (Smith 2009). The best fit obtained is $d N / d E=0.62 \times 10^{-11} E^{-1.50} \exp (-E / 14.1)$ photons $\mathrm{cm}^{-2} \mathrm{~s}^{-1} \mathrm{TeV}^{-1}$, where $E$ is the energy in TeV. This flux does not agree with that given by H.E.S.S. at a level of 2-3 standard deviations, being about a factor of three higher at $10 \mathrm{TeV}$. The authors suggest that the discrepancy could simply be due to a statistical fluctuation, or to the fact that Milagro, given its relatively poor angular resolution, integrates the signal over a larger solid angle compared with H.E.S.S., and likely detects more of the diffuse lateral tails of the extended source.

In this work we report on the observation of MGRO J1908+06 with the ARGO-YBJ detector performed during the years 2007-2011. After a brief description of the detector and a detailed presentation of the data analysis technique, we report our results concerning the extension and the energy spectrum of the source.

\section{THE ARGO-YBJ EXPERIMENT}

The ARGO-YBJ detector is located at the Yangbajing Cosmic Ray Laboratory (Tibet, China) at an altitude of $4300 \mathrm{~m}$ above sea level. It consists of a $\sim 74 \times 78 \mathrm{~m}^{2}$ carpet made of a single layer of Resistive Plate Chambers (RPCs) with $\sim 92 \%$ of active area, surrounded by a partially instrumented $(\sim 20 \%)$ area up to $\sim 100 \times 110 \mathrm{~m}^{2}$. The apparatus has a modular structure, the basic data acquisition element being a cluster $\left(5.7 \times 7.6 \mathrm{~m}^{2}\right)$ made up of 12 RPCs $\left(2.8 \times 1.25 \mathrm{~m}^{2}\right)$. The RPCs are operated in streamer mode by using a gas mixture (Ar 15\%, Isobutane 10\%, TetraFluoroEthane $75 \%$ ) suitable for high-altitude operation.

Each RPC is read by 80 strips of $6.75 \times 61.8 \mathrm{~cm}^{2}$ (the spatial pixels) that are logically organized in 10 independent pads of $55.6 \times 61.8 \mathrm{~cm}^{2}$, which are individually acquired and represent the time pixels of the detector (Aielli et al. 2006). In addition, in order to extend the dynamical range up to PeV energies, each $\mathrm{RPC}$ is equipped with two large pads $\left(139 \times 123 \mathrm{~cm}^{2}\right)$ to collect the total charge developed by the particles hitting the detector (Iacovacci et al. 2009). The full experiment is made up of 153 clusters for a total active surface of $\sim 6600 \mathrm{~m}^{2}$.

ARGO-YBJ operates in two independent acquisition modes: the shower mode and the scaler mode (Aielli et al. 2008). In this analysis we refer to the data recorded from the digital read-out in shower mode. In this mode, an electronic logic has been implemented to build an inclusive trigger based on a time correlation between the pad signals, depending on their relative distances. In this way, all the shower events that give a number of fired pads $N_{\text {pad }} \geqslant N_{\text {trig }}$ in the central carpet generate the trigger in a time window of $420 \mathrm{~ns}$. This trigger can work with high efficiency down to $N_{\text {trig }}=20$, keeping the rate of random coincidences negligible (Aloisio et al. 2004).

The time of each fired pad in a window of $2 \mu$ s around the trigger time and its location are recorded and used to reconstruct the position of the shower core and the arrival direction of the primary particle.

In order to perform the time calibration of the 18,360 pads, a software procedure based on the Characteristic Plane method (He et al. 2007) has been developed that uses the secondary particles of large vertical showers as calibration beams and iteratively reduces the differences between the measured times and the temporal fit of the shower front (Aielli et al. 2009).

The full detector has been taking data since 2007 November with the trigger condition $N_{\text {trig }}=20$ and a duty cycle of $\sim 86 \%$. The trigger rate is $\sim 3.5 \mathrm{kHz}$ with a dead time of $4 \%$.

\section{DETECTOR PERFORMANCE}

The angular resolution and the pointing accuracy of the detector have been evaluated by using the Moon shadow, i.e., the deficit of cosmic rays in the Moon direction, observed by ARGO-YBJ with a statistical significance of $\sim 9$ standard deviations per month. The shape of the shadow provides a measurement of the detector point-spread function (PSF), and its position allows the individuation of possible pointing biases.

The data have been compared with the results of a Monte Carlo simulation, which describes the propagation of cosmic rays in the Earth magnetic fields, the shower development in the atmosphere by using the CORSIKA code (Heck et al. 1998), and the detector response with a code based on the GEANT package (GEANT 1993). The PSF measured with cosmic rays has been found to be in excellent agreement with the Monte Carlo evaluation, confirming the reliability of the simulation procedure (Bartoli et al. 2011).

The angular resolution for gamma rays is evaluated by simulating the events from a gamma-ray source with a given spectrum and daily path in the sky. The results are smaller by $\sim 30 \%-40 \%$ compared with the angular resolution for cosmic rays, due to the better defined time profile of the showers. In general, the PSF for gamma rays can be described by the sum of two Gaussian distributions. For a Crab-like source, the radius of the opening angle, which optimizes the signal-to-background ratio for events with $N_{\text {pad }} \geqslant 60$ (300), is $0.86(0.44)$ and contains $\sim 50 \%$ of the signal. 
The Moon shadow has also been used to check the absolute energy calibration of the detector by studying the westward shift of the shadow due to the geomagnetic field. The observed displacement as a function of the event multiplicity $N_{\text {pad }}$ is in excellent agreement with the results of the Monte Carlo simulation. From this analysis the total absolute energy scale error, including systematic effects, is estimated to be less than 13\% (Bartoli et al. 2011).

\section{DATA ANALYSIS AND RESULTS}

At the ARGO-YBJ site MGRO J1908+06 culminates at the zenith angle of $24^{\circ}$ and is visible for $5.38 \mathrm{hr}$ day $^{-1}$ with a zenith angle less than $45^{\circ}$. The data set used in this analysis refers to the period from 2007 November to 2011 December and contains all the showers with zenith angles less than $45^{\circ}$ and $N_{\text {pad }} \geqslant 20$. The total on-source time is $6867 \mathrm{hr}$.

To study the gamma-ray emission from a source, a $16^{\circ} \times$ $16^{\circ}$ sky map in celestial coordinates (right ascension and declination) with a $0.1 \times 0.1$ bin size, centered on the source position, is filled with the detected events.

In order to extract the excess of gamma rays, the cosmic ray background has to be estimated and subtracted.

The time swapping method (Alexandreas et al. 1993) is used to evaluate the background: for each detected event, $n$ "fake" events (with $n=10$ ) are generated by replacing the original arrival time with new ones, randomly selected from an event buffer that spans a data-recording time $T$ of data taking. Changing the time, the fake events maintain the same declination of the original event, but have a different right ascension. With these events a new sky map (background map) is built, with statistics $n$ times larger than the "true" event map in order to reduce fluctuations. In order to avoid the inclusion of the source events in the background evaluation, the showers inside a circular region around the source (with a radius related to the PSF and depending on $N_{\mathrm{pad}}$ ) are excluded from the time swapping procedure. A correction of the number of swaps is made to take into account the rejected events in the source region (Fleysher et al. 2004). The value of the swapping time $T$ is $\sim 3 \mathrm{hr}$, in order to minimize the systematic effects due to the environmental parameter variations.

In order to extract the source signal, the maps are smoothed according to the detector PSF, determined by Monte Carlo simulations for different $N_{\text {pad }}$ intervals. Finally, the smoothed background map is subtracted from the smoothed event map, obtaining the "excess map" where the statistical significance $S$ of the excess for every bin is given by

$$
S=\left(N_{\text {on }}-N_{\text {off }}\right) / \sqrt{\delta N_{\text {on }}^{2}+\delta N_{\text {off }}^{2}},
$$

with $N_{\text {on }}=\Sigma_{i} N_{i} w_{i}$ and $N_{\text {off }}=\Sigma_{i} B_{i} w_{i} / n$. In these expressions $N_{i}$ and $B_{i}$ are the number of events of the $i$ th bin of the "event map" and "background map," respectively, $w_{i}$ is a weight proportional to the value of the PSF at the angular distance of the $i$ th bin, and $n$ is the number of swaps. The sum is over all the bins inside a radius $R$, chosen to contain the PSF. Since the number of events per bin is large, the fluctuations follow the Gaussian statistics, hence the errors on $N_{\text {on }}$ and $N_{\text {off }}$ are $\delta N_{\text {on }}=$ $\sqrt{\Sigma_{i} N_{i} w_{i}^{2}}$ and $\delta N_{\text {off }}=\sqrt{\Sigma_{i} B_{i} w_{i}^{2} / n^{2}}$.

In order to study the signal in different energy regions, the maps are built for eight different $N_{\text {pad }}$ intervals, namely 20-39, 40-59, 60-99, 100-199, 200-299, 300-499, 500-999, and $>1000$. These maps are then combined to have "integral maps" for different $N_{\text {pad }}$ thresholds.

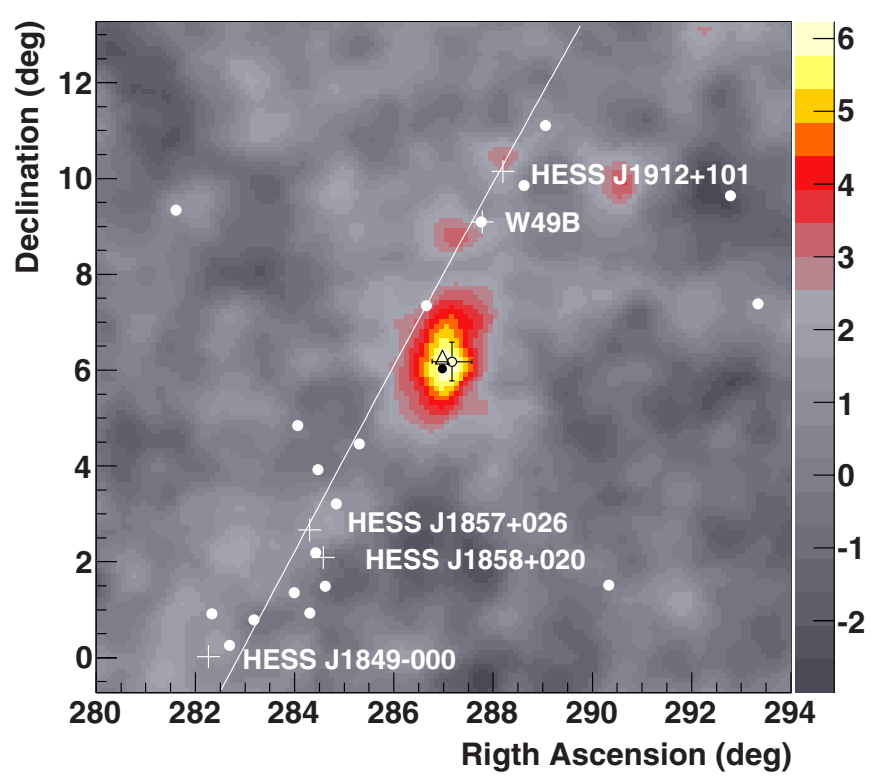

Figure 1. PSF-smoothed significance map of the MGRO J1908+06 region obtained by ARGO-YBJ for events with $N_{\text {pad }} \geqslant 60$. Open circle: position of the center of MGRO J1908+06 as measured by Milagro. The error bars give the linear sum of the statistical and systematic errors. Open triangle: centroid of HESS J1908+063. Black filled circle: Fermi pulsar PSR J1907+0602. White filled circles: Fermi gamma-ray sources, according to the second Fermi Catalogue (Nolan et al. 2012). White crosses: TeV sources detected by H.E.S.S. in the same region. The white line represents the Galactic plane.

(A color version of this figure is available in the online journal.)

Analyzing the data recorded over four years, the sky maps of the MGRO J1908+06 region show a significant excess at the source position for different $N_{\text {pad }}$ thresholds. The larger significance is given by events with $N_{\text {pad }} \geqslant 20$, with 7.3 standard deviations. When $N_{\text {pad }}$ increases, the significance decreases. For $N_{\text {pad }}>1000$, no signal is present.

The distributions of the significances outside the source region follow a standard normal distribution, showing the correctness of the background evaluation procedure.

As will be discussed in Section 4.3, the signal with $N_{\text {pad }}=$ 20-59 is largely affected by the Galactic diffuse gamma-ray flux, and only events with $N_{\text {pad }} \geqslant 60$ will be used in the study of the source morphology and flux. Figure 1 shows the significance map for events with $N_{\text {pad }} \geqslant 60$, where the source signal reaches 6.2 standard deviations.

Studying the source in time intervals of one year, the annual excess rates are consistent with the total average rate, indicating that the gamma-ray flux from MGRO J1908+06 is likely due to a steady emission.

\subsection{Source Position and Extension}

To evaluate the position and extension of the source, the events with $N_{\text {pad }} \geqslant 60$ are used. We assume a source shape described by a symmetrical two-dimensional Gaussian function with rms $\sigma_{\text {ext }}$. Fitting the non-smoothed excess map to a function given by the convolution of the above Gaussian and the detector PSF, we found the best-fit position at R.A. $=19^{\mathrm{h}} 08^{\mathrm{m}} 1^{\mathrm{s}}$ and decl. $=6^{\circ} 24^{\prime}$, with a statistical error of $12^{\prime}$ and a systematic error of $6^{\prime}$ per axis. The position found is consistent with the Milagro measurement and with the centroid of HESS J1908+063 (R.A. $=19^{\mathrm{h}} 07^{\mathrm{m}} 54^{\mathrm{s}}$ and decl. $=6^{\circ} 16^{\prime} 7^{\prime \prime}$, with a statistical error of 2.4 and a systematic error of $20^{\prime \prime}$ per axis).

The value of $\sigma_{\text {ext }}$ that best fits the data is $0.49 \pm 0.22$, and is consistent with the H.E.S.S. estimation of 0.34 . 


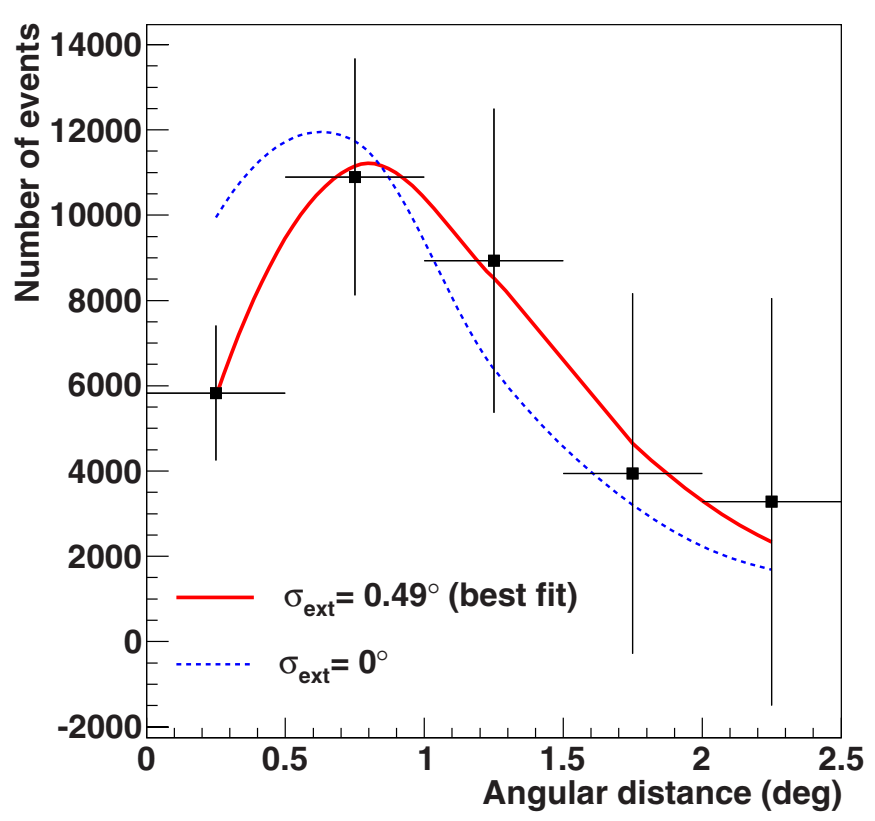

Figure 2. Number of excess events with $N_{\text {pad }} \geqslant 60$ as a function of the angular distance from the best-fit centroid position compared to the expected distributions for different source extensions.

(A color version of this figure is available in the online journal.)

Figure 2 shows the distribution of the angular distance from the best-fit centroid position compared to the simulated distributions corresponding to the extensions $\sigma_{\text {ext }}=0.49$ and $\sigma_{\text {ext }}=0^{\circ}$. The two curves are normalized to the same number of excess events.

\subsection{Energy Spectrum}

In order to study the energy dependence of the signal, the events are divided into different subsets according to the number of hit pads, and a sky map is built for each subset. For this analysis we define four intervals: $N_{\text {pad }}=20-59,60-199$, 200-499, and $N_{\text {pad }} \geqslant 500$. The intervals have been chosen in order to have a signal of comparable statistical significance.

For the spectrum evaluation we assume a power-law dependence of $d N / d E=K E^{-\gamma}$. The values of $K$ and $\gamma$ are derived by comparing the number of the excess events detected in each of the previously defined $N_{\text {pad }}$ intervals with the corresponding ones given by simulations assuming a set of test spectra. The reliability of this procedure has been tested by studying the Crab Nebula signal (Aielli et al. 2010).

For each $N_{\text {pad }}$ interval, the number of excess events is obtained by integrating the sky map around the source position up to a distance $\psi_{\max }$, where $\psi_{\max }$ is the radius of the opening angle that maximizes the signal to background ratio. The value of $\psi_{\max }$ depends on the source extension $\sigma_{\text {ext }}$ and on the detector PSF, and is provided by simulations. For the extension we use the value $\sigma_{\text {ext }}=0.49$, according to our measurement. On the other hand, the PSF for a given $N_{\text {pad }}$ interval is not precisely determined, since it depends on both the detector characteristics and the spectrum index $\gamma$, which is unknown. To solve this "circular" problem, an iterative procedure has been applied.

First, an initial index $\gamma=2.5$ is assumed, and the corresponding values of $\psi_{\max }$ for every $N_{\text {pad }}$ interval are determined via simulations. The number of events observed in $\psi_{\max }$ is then used to evaluate a new spectral slope $\gamma$, which is returned to the first step, to calculate a new set of $\psi_{\max }$, and so on. Given the relatively weak dependence of the PSF on $\gamma$, a small number of iterations is sufficient to terminate the process successfully and provide the parameters of the best-fit spectrum.

\subsection{Contribution from Diffuse Flux}

Since the source is located on the Galactic plane, the observed flux could be affected by the diffuse gamma-ray emission produced by cosmic rays interacting with the matter and the radiation fields of the Galaxy. Given the relatively large opening angles used in the measurement, the photons from the diffuse radiation falling in the observational window of MGRO J1908+06 could artificially increase the flux detected from the source direction. The amount of this contribution can be evaluated by analyzing the data collected from the Galactic plane region close to the source.

The flux of very high energy Galactic gamma rays in the region of MGRO J1908+06 (Galactic coordinates $l=40.39$ and $b=-0.79)$ is poorly known.

The first evidence of a diffuse Galactic emission at $\mathrm{TeV}$ energies has been reported by the Milagro detector (Atkins et al. 2005). A significant dependence of the flux on the Galactic latitude and longitude has been found in a later analysis with events of median energy $15 \mathrm{TeV}$ (Abdo et al. 2008). The same paper reports the expected energy spectrum for two different sectors of the Galactic plane for energies from $10 \mathrm{keV}$ to $100 \mathrm{TeV}$, according to the GALPROP model (Strong et al. 2000; Porter et al. 2008), "optimized" to fit the measurements by EGRET in the $40 \mathrm{MeV}-10 \mathrm{GeV}$ energy range and by Milagro at $15 \mathrm{TeV}$. Concerning the MGRO J1908+06 region, the expected average flux at $1 \mathrm{TeV}$ in the area of Galactic coordinates $l \in\left[30^{\circ}, 65^{\circ}\right]$ and $b \in\left[-2^{\circ}, 2^{\circ}\right]$ is $\sim 2 \times 10^{-9}$ photons $\mathrm{TeV}^{-1} \mathrm{~cm}^{-2} \mathrm{~s}^{-1} \mathrm{sr}^{-1}$.

A preliminary flux measurement at energies $E>300 \mathrm{GeV}$, obtained with the ARGO-YBJ data, is reported by Ma (2011), who derives the average gamma-ray spectrum for $l \in\left[25^{\circ}, 65^{\circ}\right]$ and $b \in\left[-2^{\circ}, 2^{\circ}\right]$. This estimate is lower but still consistent with the expectation of the above model.

For our purposes, given the variation of the emission along the Galactic plane and its strong dependence on the latitude, it is preferable to evaluate the diffuse flux in a restricted region adjacent to the source position.

We consider two sky regions, $\mathrm{L}_{1}$ and $\mathrm{L}_{2}$, with a size of $\Delta l=5^{\circ}$ and $\Delta b=2 \times \psi_{\max }$ (where $\psi_{\max }$ depends on the $N_{\text {pad }}$ interval) whose centers have the same latitude of MGRO $\mathrm{J} 1908+06$ and are located at both sides of the source at a longitudinal distance of 5.5 .

Analyzing the ARGO-YBJ data, a global excess of statistical significance that is 3.0 standard deviations over the cosmic ray background is observed in $\mathrm{L}_{1}+\mathrm{L}_{2}$ for events with $N_{\text {pad }}>$ 20. This excess is thought to be due to the diffuse Galactic emission plus the contribution of five gamma-ray sources discovered by H.E.S.S., namely HESS J1912+101 (Aharonian et al. 2008b), W49B (Brun et al. 2011), HESS J1857+026 and HESS J1858+020 (Aharonian et al. 2008a), and HESS J1849000 (Terrier et al. 2008).

The individual fluxes of these objects are below the ARGOYBJ sensitivity, while the total flux is $(29 \pm 3) \%$ that of the Crab Nebula at $1 \mathrm{TeV}$ (Vernetto 2011). In particular, HESS J1912+101 and HESS J1857+026 have a flux that is $\sim 10 \%$ and $\sim 17 \%$ that of the Crab Nebula, respectively. The number of events from these sources expected to fall in $\mathrm{L}_{1}$ and $L_{2}$ is evaluated via simulations, using the fluxes measured by H.E.S.S., and gives a global contribution of $(40 \pm 14) \%$ 


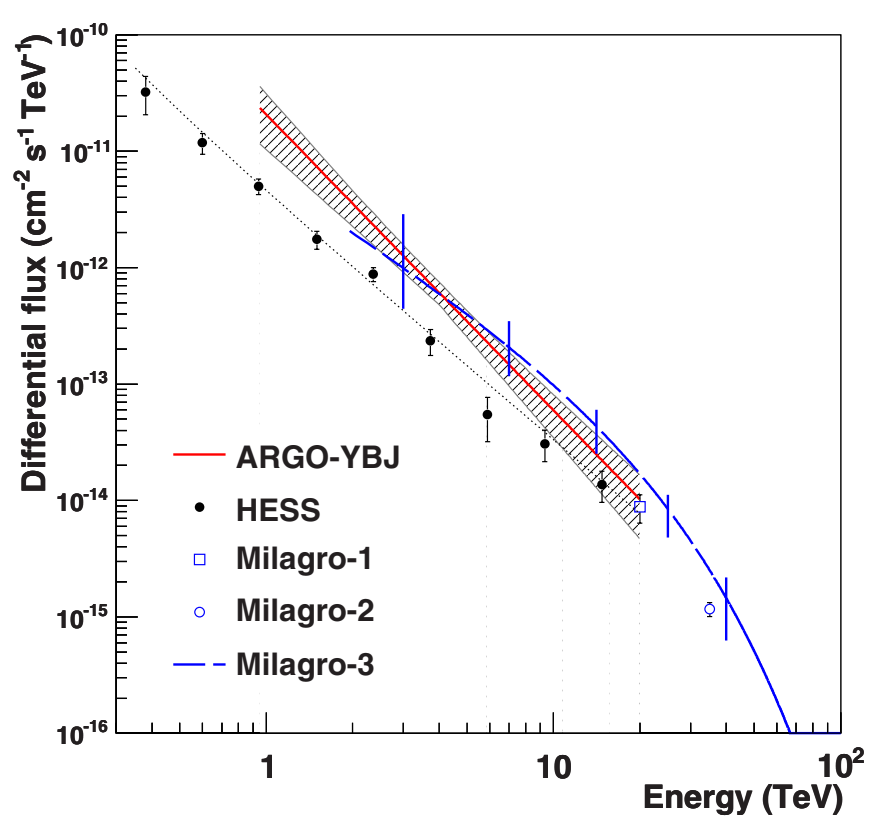

Figure 3. Gamma-ray flux from MGRO J1908+06 measured by different detectors. ARGO-YBJ: the continuous line (red in the online version) is the best fit to the data. The dashed area represents a one standard deviation error. H.E.S.S.: the dotted line is the best fit to the points (Aharonian et al. 2009). Milagro 1: flux value assuming a spectrum $\propto E^{-2.3}$ (Abdo et al. 2007). Milagro 2: flux value assuming a spectrum $\propto E^{-2.6}$ (Abdo et al. 2009b). Milagro 3: the dashed line (blue in the online version) is the spectrum fit according to Smith (2009) and the vertical lines are the errors (at one standard deviation) for some values of the energy. The plotted errors are purely statistical for all the detectors. (A color version of this figure is available in the online journal.)

to the observed diffuse excess. After the subtraction of this contribution, taking into account the different exposures of $\mathrm{L}_{1}$ and $L_{2}$, we evaluate the number of events due to the diffuse emission expected to fall into the observational window of MGRO J1908+06.

We found that the ratio between the number of events expected from the diffuse emission and those observed from the source direction is $R_{d}=0.33 \pm 0.18$ for showers with $N_{\text {pad }}=20-59$, and $R_{d}<0.15$ (at one sigma level) for showers with $N_{\text {pad }} \geqslant 60$.

As a comparison, the values derived by using the "optimized" GALPROP diffuse emission model given by Abdo et al. (2008) are $R_{d}=0.57$ for $N_{\text {pad }}=20-59$, and $R_{d}=0.23$ for $N_{\text {pad }} \geqslant 60$. These values are larger than those obtained with the ARGO-YBJ data. It should be noted, however, that the above model is based on a measurement by Milagro that does not take into account all the gamma-ray sources located in the studied region and could overestimate the diffuse flux.

The larger contribution of the diffuse emission for $N_{\text {pad }}=$ 20-59 is due to the wider opening angle used in this interval $\left(\psi_{\max }=2.0\right)$. Because of these estimates, to avoid a possible large systematic effect in the flux evaluation, we restrict our spectral analysis to the events with $N_{\text {pad }} \geqslant 60$.

Performing the procedure described in Section 4.2, we fit the data of the three intervals $N_{\mathrm{pad}}=60-199,200-499$, and $N_{\text {pad }} \geqslant 500$. The best-fit spectrum obtained is $d N / d E=$ $6.1 \pm 1.4 \times 10^{-13}(E / 4 \mathrm{TeV})^{-2.54 \pm 0.36}$ photons $\mathrm{cm}^{-2} \mathrm{~s}^{-1} \mathrm{TeV}^{-1}$, valid in the energy region $1-20 \mathrm{TeV}$. The median energies corresponding to the three $N_{\text {pad }}$ intervals are 2.4, 5.1, and $12.8 \mathrm{TeV}$, respectively.

As a comparison, if we do not exclude the data with $N_{\mathrm{pad}}=$ $20-59$, the best-fit spectrum is $d N / d E=1.36 \pm 0.29 \times 10^{-12}$
$(E / 3 \mathrm{TeV})^{-2.65 \pm 0.25}$ photons $\mathrm{cm}^{-2} \mathrm{~s}^{-1} \mathrm{TeV}^{-1}$, which gives a flux that is $21 \%$ higher at $E=1 \mathrm{TeV}$.

In addition to the statistical errors and the systematics due to the diffuse contribution discussed above, our measurement could be affected by an additional systematic error mainly due to the background evaluation, the absolute energy scale determination, the pointing accuracy, environmental effects, and the Monte Carlo simulations for a global effect that we estimate to be $<30 \%$ (Aielli et al. 2010).

In the case of an extended source, a possible further cause of systematics could be the uncertainty in the extension, and the consequent use of an incorrect opening angle to extract the signal. Therefore, we have also evaluated the spectrum assuming $\sigma_{\text {ext }}=0.34$, as measured by H.E.S.S. The resulting flux differs from the previous one by less than $5 \%$ in the whole energy range considered in the analysis.

The obtained spectrum is shown in Figure 3, together with those reported by H.E.S.S. and Milagro. The flux is significantly higher than that given by H.E.S.S. in the $1-10 \mathrm{TeV}$ energy range, but is consistent with the Milagro spectrum (Smith 2009). The hard spectrum with an exponential cutoff obtained by Milagro produces a worse, but still acceptable, fit to our data. Given the reduced significance of the excess at high energies, we are not able to constrain the shape of the spectrum above $10 \mathrm{TeV}$ and definitively rule out a high-energy cutoff.

\section{DISCUSSION AND CONCLUSIONS}

The gamma-ray source MGRO J1908+06 has been studied by ARGO-YBJ analyzing $\sim 4$ years of data. An excess with a significance of 6.2 standard deviations is observed in a position consistent with previous measurements by Milagro and H.E.S.S. The peak of the signal occurs at R.A. $=19^{\mathrm{h}} 08^{\mathrm{m}} 1^{\mathrm{s}}$ and decl. $=6^{\circ} 24^{\prime}$ (with statistical and systematic errors of $\sim 0.2$ and 0.1 per axis, respectively) and lies at a distance of $22^{\prime}$ from PSR J1907+0602, consistent with the pulsar location within the measurement error.

The signal is due to emission from an extended region. After taking into account the detector PSF, the extension of the source is found to be $\sigma_{\text {ext }}=0.49 \pm 0.22$.

The photon spectrum in the range of $1-20 \mathrm{TeV}$ follows a simple power law with a spectral index of $2.54 \pm 0.36$, though a harder spectrum with a high-energy cutoff cannot be ruled out.

The spectrum is found to be consistent with the Milagro result (Smith 2009) but not with the H.E.S.S. best fit in the 1-10 energy range, where the flux measured by ARGO-YBJ at $4 \mathrm{TeV}$ is a factor of 2.6 larger. At $\sim 20 \mathrm{TeV}$ the ARGO-YBJ, H.E.S.S., and Milagro fluxes are consistent within the errors and are also in agreement with the first Milagro measurement (Abdo et al. 2007).

Since a contribution to this measurement is expected from the Galactic diffuse emission, data from the two sky regions located at both sides of the source and centered at the same latitude have been used to determine this contamination. According to this estimate, the diffuse Galactic gamma-ray emission is expected to contribute to the signal above $1 \mathrm{TeV}$ for less than $\sim 15 \%$, and cannot account for the observed disagreement.

Since the difference with H.E.S.S. is at the level of 2.5 standard deviations, the discrepancy could be simply due to statistical fluctuations or the combination of statistical and systematic uncertainties. However, these latter causes have been accurately studied, giving a global error of less than $30 \%$ on the flux. Indeed, the spectrum of the Crab Nebula obtained by 
ARGO-YBJ is in good agreement with the Cherenkov detector measurements (Aielli et al. 2010; Vernetto 2011). The extension of the source should not give an additional systematic error to explain the observed difference.

On the other hand, a similar discrepancy is found in the observation of the extended source MGRO J2031+41, located in the Cygnus region, for which ARGO-YBJ (Bartoli et al. 2012) and Milagro (Abdo et al. 2012) report a flux significantly larger than that measured by the Cherenkov Telescopes MAGIC and HEGRA.

In principle, one cannot exclude the possibility of a flux variation as the origin of the observed disagreement among the detectors. Milagro, H.E.S.S., and ARGO-YBJ data have been recorded in different periods. Milagro integrates over 7 years (2000 July-2007 November) while the total H.E.S.S. data set only amounts to $27 \mathrm{hr}$ of sparse observations during 2005-2007, before the ARGO-YBJ measurement. However, a possible flux variation seems unlikely, since the average fluxes measured by Milagro and ARGO-YBJ in two contiguous periods covering a total time of 11 years are consistent.

Moreover, it should be noted that if MGRO J1908+06 is the pulsar wind nebula associated with PSR J1907+0602, the gamma-ray emission originates from a region whose size has been estimated to be $\geqslant 40 \mathrm{pc}$ (Abdo et al. 2010), implying that the variation timescale cannot be less than $\sim 130$ years, unless relativistic beaming effects are present.

In conclusion, MGRO J1908+06 is observed by ARGO-YBJ as a stable extended source, likely the TeV nebula of PSR $\mathrm{J} 1907+0602$, with a flux at $1 \mathrm{TeV}$ that is $\sim 67 \%$ that of the Crab Nebula. Assuming a distance of $3.2 \mathrm{kpc}$, the integrated luminosity above $1 \mathrm{TeV}$ is $\sim 1.8$ times that of the Crab Nebula, making MGRO J1908+06 one of the most luminous Galactic gamma-ray sources at $\mathrm{TeV}$ energies.

This work is supported in China by NSFC (No. 10120130794), the Chinese Ministry of Science and Technology, the Chinese Academy of Science, the Key Laboratory of Particle Astrophysics, CAS, and in Italy by the Istituto Nazionale di Fisica Nucleare (INFN).

We also acknowledge the essential support of W. Y. Chen, G. Yang, X. F. Yuan, C. Y. Zhao, R. Assiro, B. Biondo, S. Bricola, F. Budano, A. Corvaglia, B. D’Aquino, R. Esposito, A. Innocente, A. Mangano, E. Pastori, C. Pinto, E. Reali, F. Taurino, and A. Zerbini in the installation, debugging, and maintenance of the detector.

\section{REFERENCES}

Abdo, A. A., Abeysekara, U., Allen, B. T., et al. 2012, ApJ, 753, 159

Abdo, A. A., Ackermann, M., Ajello, M., et al. 2009a, ApJS, 183, 46

Abdo, A. A., Ackermann, M., Ajello, M., et al. 2010, ApJ, 711, 64

Abdo, A. A., Allen, B., Aune, T., et al. 2008, ApJ, 688, L1078

Abdo, A. A., Allen, B. T., Aune, T., et al. 2009b, ApJ, 700, L127

Abdo, A. A., Allen, B., Berley, D., et al. 2007, ApJ, 664, L91

Aharonian, F., Akhperjanian, A. G., Anton, G., et al. 2009, A\&A, 499, 723

Aharonian, F., Akhperjanian, A. G., Barres de Almeida, U., et al. 2008a, A\&A, 477,353

Aharonian, F., Akhperjanian, A. G., Barres de Almeida, U., et al. 2008b, A\&A, 484,435

Aielli, G., Assiro, R., Bacci, C., et al. 2006, Nucl. Instrum. Methods Phys. Res. A, 562,92

Aielli, G., Bacci, C., Barone, F., et al. 2008, Astropart. Phys., 30, 85

Aielli, G., Bacci, C., Bartoli, B., et al. 2009, Astropart. Phys., 30, 287

Aielli, G., Bacci, C., Bartoli, B., et al. 2010, ApJ, 714, L208

Alexandreas, D. E., Berley, D., Biller, S., et al. 1993, Nucl. Instrum. Methods Phys. Res. A, 328, 570

Aloisio, A., Branchini, P., Catalanotti, S., et al. 2004, IEEE Trans. Nucl. Sci., 51,1835

Amenomori, M., Bi, X. J., Chen, D., et al. 2010, ApJ, 709, L6

Atkins, R., Benbow, W., Berley, D., et al. 2005, Phys. Rev. Lett., 95, 251103

Bartoli, B., Bernardini, P., Bi, X. J., et al. 2011, Phys. Rev. D, 84, 022003

Bartoli, B., Bernardini, P., Bi, X. J., et al. 2012, ApJ, 745, L22

Brun, F., De Naurois, M., Hofmann, W., et al. 2011, in Proc. 25th Texas Symposium on Relativistic Astrophysics (available at arXiv:1104.5003v1)

Fleysher, R., Fleysher, L., Nemethy, P., \& Mincer, A. I. 2004, ApJ, 603, 355

GEANT. 1993, Detector Description and Simulation Tool 1993, CERN Program Library, W5013, http://wwwasd.web.cern.ch/wwwasd/geant/

Heck, D., Knapp, J., Capdevielle, J. N., Shatz, G., \& Thouw, T. 1998, Forschungszentrum Karlsruhe Report No. FZKA 6019

He, H. H., Bernardini, P., Calabrese Melcarne, A. K., \& Chen, S. Z. 2007, Astropart. Phys., 27, 528

Iacovacci, M., Corvaglia, A., Creti, P., et al. 2009, in Proc. 31st Int. Cosmic Ray Conf., Lodz, Poland (available at http://icrc2009.uni.lodz.pl/proc/html/)

Ma, L. L. 2011, in Proc. 32nd Int. Cosmic Ray Conf., Beijing, China (available at http://www.ihep.ac.cn/english/conference/icrc2011/paper/), Vol. 7, 2

Nolan, P. L., Abdo, A. A., Ackermann, M., et al. 2012, ApJS, 199, 31

Porter, T. A., Moskalenko, I. V., Strong, A. W., Orlando, E., \& Bouchet, L. 2008, ApJ, 682, 400

Smith, A. J. 2009, in Proc. Fermi Symposium, Conf Proceedings C091122, http://www.slac.stanford.edu/econf/C0911022/

Strong, A. W., Moskalenko, I. V., \& Reimer, O. 2000, ApJ, 537, 763

Terrier, R., Mattana, F., Djannati-Atai, A., et al. 2008, in AIP Conf. Proc. 1085, Proc. 4th Int. Meeting on High Energy Gamma-Ray Astronomy, ed. F. A. Aharonian, W. Hoffman, \& F. M. Rieger (Melville, NY: AIP), 312

Vernetto, S. 2011, in Proc. 32nd Int. Cosmic Ray Conf., Beijing, China (available at //www.ihep.ac.cn/english/conference/icrc2011/paper/), Vol. 7, 126

Ward, J. E. 2008, in AIP Conf. Proc. 1085, High energy Gamma-Ray Astronomy, ed. F. A. Aharonian, W. Hofmann, \& F. Rieger (Melville, NY: AIP), 301

Zhang, J. L. 2003, in Proc. 28th Int. Cosmic Ray Conf. 4, ed. T. Kajita et al. (Tokyo: Universal Academy Press, Inc.), 2405 